\title{
Integrating an Interprofessional Education Model at a Private University
}

\author{
Ramona Ann Parker ${ }^{1}$, Helmut Gottlieb ${ }^{2}$, Daniel G Dominguez ${ }^{3}$, Patricia C. Sanchez-Diaz ${ }^{4} \&$ Mary Elaine Jones $^{1}$ \\ ${ }^{1}$ Department of Nursing, University of the Incarnate Word, USA \\ ${ }^{2}$ Feik School of Pharmacy, University of the Incarnate Word, USA \\ ${ }^{3}$ HEB School of Business Administration, University of the Incarnate Word, USA \\ ${ }^{4}$ Rosenberg School of Optometry, University of the Incarnate Word, USA \\ Correspondence: Ramona Ann Parker, Department of Nursing, University of the Incarnate Word, USA. E-mail: \\ parker@uiwtx.edu
}

Received: June 29, 2015

Accepted: July 21, 2015

Online Published: July 24, 2015

doi:10.5430/ijhe.v4n3p112

URL: http://dx.doi.org/10.5430/ijhe.v4n3p112

\begin{abstract}
In 2012, a private University in South Texas sought to prepare eight cohorts of 25 nursing, optometry, pharmacy, physical therapy, and health care administration students with an interprofessional education activity as a model for collaborative learning. The two semester interprofessional activity used a blended model (Blackboard Learn ${ }^{\circledR}$, face-to-face and simulated experiences) to teach the core competencies of Interprofessional Education and Collaborative Practice (IPECP). The organizing framework for the curriculum sequence included concepts of health promotion, prevention and intervention embedded in the Web of Causation (Primary, Secondary, Tertiary care). One hundred ninety-eight students participated in the interprofessional educational activity. Several of the disciplines used multiple methods to invite students to participate in this voluntary effort. One discipline provided a means by which students could select this activity as an elective and another integrated the activity into an existing course.

While not completely new, transforming the way pre-licensed students and faculty in health professions education experience clinical and didactic environments is an imperative. Interprofessional education and clinical practice must be an intentional preparation that needs and requires support from university administration for successful implementation and sustainability.
\end{abstract}

Keywords: Interprofessional education, Higher education, Collaboration

\section{Introduction}

The Institute of Medicine (IOM) identified interdisciplinary education and practice for the health professions as part of an educator's obligation both in the classroom and in clinical practice (Institute of Medicine, 1972). D'Amour and Oandansan (2005) introduced the concept of interprofessionality in which students, faculty, and direct care organizations engage in interprofessional education and collaborative practice in order to promote good patient outcomes. Benner (2011) further suggests that interprofessional collaboration creates and elucidates civic professionalism. Civic professionalism is manifest when an organization accepts the tacit inter-relatedness of each profession's knowledge, skills and abilities. Once civic professionalism, which is not inherent, takes root, individual health professions refrain from practicing in a silo and begin promoting and engaging in collaborative behaviors and actions that positively influence the health system.

A growing number of private and public organizations support and encourage academicians and practitioners to depart from a culture of silo-ism and embrace a culture of collaborative partnerships (Interprofessional Education and Collaborative Practice, 2011; Josiah Macy Foundation, 2013; Robert Wood Johnson Foundation, 2011). Further, a number of health professional education accrediting bodies, e.g., nursing (Interprofessional Education and Collaborative Practice, 2011), pharmacy (Accreditation Council for Pharmacy Education, 2011), physical therapy (Commission on Accreditation of Physical Therapy Education, 2013), optometry (Accreditation Council on Optometric Education, 2009), and health administration (Commission on Accreditation of Healthcare Management Education, 2015), include language mandating student involvement in Interprofessional Education (IPE) activities. As such, students enrolled in these accredited programs are required to engage in IPE activities.

However, pedagogies that reshape conventional approaches to health professional education remain elusive as evidence of strategies that positively affect attitudes and behaviors toward interprofessionality and collaborative 
practice teams are few (Thistlewaite, 2012; Lapkin, Levett-Jones \& Gilligan, 2013). This paper describes the steps to implement an interprofessional educational experience with five health professional schools and the challenges to sustaining interprofessional activities at the University level.

\subsection{Background}

The setting for this initiative was a medium-sized, private, faith-based University located in the southwest United States. The University is designated as a Hispanic-Serving liberal arts institution with a student population of 9,500. The focus of the University's growth over the past few years has been on health professions by adding four new professional schools in the past nine years. The University's receipt of federal funding in 2012 from the Division of Nursing, Health Resources and Services Administration (HRSA) created an opportunity to integrate the concepts of interprofessional education and collaborative practice into the didactic and clinical components of five programs: nursing, pharmacy, physical therapy, optometry and health administration. These health professional programs were selected because of national trends toward interprofessional education in healthcare professional programs; more specifically the schools of nursing and pharmacy accrediting bodies have included interprofessionality as part of criteria for accreditation as previously described. The grant allowed for the development and implementation of a broad Interprofessional Education and Collaborative Practice initiative over a three-year funding cycle focusing on an underserved diabetic population. This demographic was chosen since a number of patients suffer from uncontrollable disease and would most likely require input from all the health professions involved in this project.

\section{Method}

\subsection{Engagement of Faculty}

The overarching philosophy to prepare "collaborative-practice ready" students required both education and practice in a real-world setting. Therefore, students participated in educational experiences to introduce essential knowledge and skills for effective formation of interprofessional relationships before engaging in direct patient care as a member of a clinical team. Deans from participating schools appointed two faculty members each to create a thirteen-member IPECP faculty committee, which was further divided into two teams: an educational team and a practice team. The educational team members $(n=6)$ developed an interprofessional educational experience to prepare students prior to exposure to an interprofessional clinical experience. The faculty practice team $(n=7)$ created and implemented a collaborative practice model for faculty and students in a clinical setting. This paper describes the implementation of the educational experience; the collaborative practice model for students is described elsewhere (Cauthon et al., 2015).

\subsection{Educational Implementation}

The educational team used the Core Competencies for Interprofessional Collaborative Practice (IOM, 1972) as the conceptual framework for development of the educational activity student outcomes (see Table 1). The IPE activity was 12-14 hours ( 1 credit hour) in length. This credit hour designation allowed nursing to integrate the IPE activity into an existing course and pharmacy to provide a stand-alone required elective for students from their programs. 
Table 1. Interprofessional educational student outcome objectives

\begin{tabular}{|c|c|}
\hline Objective & Competency Domains \\
\hline \multirow[t]{3}{*}{ Effectively collaborate with other healthcare professionals } & Teams/Teamwork \\
\hline & Interprofessional Communication \\
\hline & Roles and Responsibilities \\
\hline \multirow{4}{*}{$\begin{array}{l}\text { Discuss current health care issues and the importance of healthcare } \\
\text { teams in addressing the Institute for Healthcare Improvement's Triple } \\
\text { Aim of improving the patient experience of care (including quality of } \\
\text { care and patient satisfaction); improving the health of populations; and } \\
\text { reducing the per capita cost of health care }\end{array}$} & Teams/Teamwork \\
\hline & Interprofessional Communication \\
\hline & Roles and Responsibilities \\
\hline & Values/Ethics \\
\hline \multirow{2}{*}{$\begin{array}{l}\text { Develop trusting, working relationships with other professionals } \\
\text { involved in assessing and addressing the health care needs of patients } \\
\text { and populations served }\end{array}$} & Interprofessional Communication \\
\hline & Roles and Responsibilities \\
\hline \multirow{4}{*}{$\begin{array}{l}\text { Demonstrate knowledge, skills, and abilities which are common to all } \\
\text { healthcare professions and that underpin the delivery of quality patient } \\
\text { and population services }\end{array}$} & Teams/Teamwork \\
\hline & Interprofessional Communication \\
\hline & Roles and Responsibilities \\
\hline & Values/Ethics \\
\hline
\end{tabular}

To meet the needs of the different health professions, the educational sequence of activities was designed in a six-week blended format consisting of three online activities and three face-to-face meetings in succession. Online activities were managed using the learning management system Blackboard Learn ${ }^{\circledR}$ for IPE announcements, entre to online activities, online discussions, and as a repository for IPE reference articles. Additionally, the education team reviewed and evaluated multiple resources in order to meet the IPE blended activity outcomes. Modules developed by the Institute for Healthcare Improvement Open School (IHI) were especially effective at delivering content congruent with the four IPE domains. Selected Technology, Entertainment, Design (TED) videos supported the IHI modules; both online tools prepared students for content reinforced during the face-to-face sessions. Consequently, each online BlackBoard Learn ${ }^{\circledR}$ module created included the following: an IHI module, supplementary TED talk videos and reflective questions posted on discussion board.

Face-to-face meetings typically lasted for two hours and were conducted in the evenings after normal school hours. This activity included team formation exercises and case-based scenarios analogous to patient profiles frequently encountered in the selected primary care setting. Not all healthcare professional students or faculty were familiar with problem-based learning; students were introduced to this educational approach during face-to face sessions, thus providing familiarity to the concept prior to the last face-to-face simulation experience.

During the last face-to-face session, "actors" (nursing faculty) portrayed a standardized patient case that was provided to the students at the time of the simulation exercise. Students were placed in teams representing all five professions and given 15 minutes to review the case and develop a care plan. After the pre-work, students were escorted to the simulation area and given 20 minutes to interact with the "patient." Following the simulation exercise, students debriefed for 15-20 minutes with a faculty member facilitating the discussion. After the debrief session, students were allowed to "redo" the simulated exercise with a different actor playing the same role. Four groups of five student-teams participated simultaneously in the simulated exercise. Each student team experienced the same case with different "actors", while faculty observed in a separate room through real-time video feeds.

Faculty team members participated in a 6-8 hour workshop over 2 days on how to facilitate small student groups. In addition, faculty who volunteered to play the role of the "patient" were provided the case one-week prior to the exercise. They were briefed on what was expected of them, which included "taking off their faculty hat" and embracing the role of a patient. The actors were given free rein to enact the patient personality of their choice. For example, actors could choose to be a secluded defensive patient or an outspoken one.

\section{Results}

Implementing an interprofessional educational program across five disciplines required problem-solving skills, creativity, and patience. The following section describes the unique challenges faced by each health profession and a discussion of issues that were university-wide. Table 2 provides the characteristics of the student group. 
Table 2. Student characteristics $(\mathrm{N}=198)$

\begin{tabular}{llll}
\hline & $\begin{array}{l}\text { Total } \\
(\mathrm{N}=198)\end{array}$ & $\begin{array}{l}\text { Male } \\
(\mathrm{n}=45)\end{array}$ & $\begin{array}{l}\text { Female } \\
(\mathrm{n}=153)\end{array}$ \\
\hline Age* $^{\mathrm{X}=27.14}$ & $\mathrm{X}=27.57$ & $\mathrm{X}=27.01$ \\
$\mathrm{SD}=6.159$ & $\mathrm{SD}=5.777$ & $\mathrm{SD}=6.281$ \\
$\mathrm{Range}=21-53$ & $\mathrm{Range}=21-45$ & $\mathrm{Range}=21-53$ \\
Race/Ethnicity & & $5(11.1 \%)$ & $15(9.8 \%)$ \\
African American & $20(10.1 \%)$ & $10(22.2 \%)$ & $26(17 \%)$ \\
Asian/Pacific Islander & $36(18.2 \%)$ & $15(33.3 \%)$ & $58(37.9 \%)$ \\
Caucasian & $73(36.9 \%)$ & $14(31.1 \%)$ & $43(28.1 \%)$ \\
Hispanic & $57(28.8 \%)$ & -- & $10(6.5 \%)$ \\
Other & $10(5 \%)$ & $1(2.2 \%)$ & $1(0.7 \%)$ \\
Unreported & $2(1 \%)$ & & \\
Degree Program & & $12(26.7 \%)$ & $23(15 \%)$ \\
Healthcare Administration & $35(17.7 \%)$ & $6(13.3 \%)$ & $40(26.1 \%)$ \\
Nursing & $46(23.2 \%)$ & $9(20.0 \%)$ & $19(12.4 \%)$ \\
Optometry & $28(14.1 \%)$ & $10(22.22 \%)$ & $57(37.3 \%)$ \\
Pharmacy & $67(33.83 \%)$ & $8(17.8 \%)$ & $14(9.2 \%)$ \\
Physical Therapy & $22(11.11 \%)$ & &
\end{tabular}

$(*) \mathrm{X}=$ average; $\mathrm{SD}=$ Standard Deviation

\subsection{Health Professional Schools}

\subsubsection{Nursing}

The School of Nursing chose to integrate the IPE content and activities within an existing 3-hour course in the undergraduate and graduate programs. Formal and informal discussions with the undergraduate and graduate faculty curriculum committee chairs and review of course outcomes to assure congruency, were followed by a formal proposal to the Dean and School of Nursing curriculum committee for approval. Students in good academic standing, who demonstrated strong leadership skills were invited to participate and provided an application.

The IPE educational component for the undergraduate students occurred in the fourth semester of a five semester nursing program. The clinical experience was conducted in the fifth semester and accounted for 24 hours of 128 clinical hours required in the clinical course. Graduate student nurses (Clinical Nurse Specialist) were invited by the course faculty to participate in the IPE educational experience. The same format used for the undergraduate students was followed with graduate students. Course faculty assured students in both programs that IPE activities would not serve as additional course requirements, but would involve an equal substitution based on clinical/course outcomes. For example, one reflective paper concentrating on the students' IPE experience substituted for one required paper in the course.

\subsubsection{Pharmacy}

A class announcement for the third year students (P3) described the IPE initiative as an opportunity to meet the elective requirement for graduation. Third year students were chosen because they already had one full year of pharmacotherapeutic coursework, patient assessment experience, and would have completed their cardiovascular and endocrine module before their interprofessional practice experience. Third year students were the most appropriate candidates for the interprofessional experience because (1) they had the minimum base-knowledge and experience in evaluating patient cases and history, (2) their schedules were more consistent with those of students in the other schools; and (3) they had been exposed to drug-literature, patient therapy and had already been trained in optimizing the patient's therapy.

Students notified the pharmacy project coordinator of their intention to participate; priority was given to students on a first come, first served basis. If students had a schedule conflict with courses or required practicums, attempts were 
made to reassign them to the next cohort of IPE students.

\subsubsection{Optometry}

The interprofessional educational experience was incorporated within the pre-existing Clinical Internship courses that students take during their third and fourth years. The IPE committee representatives from optometry sent an informative recruitment email to the entire third and fourth year classes. The IPE faculty representatives selected the optometry students who volunteered to participate in the IPE initiative on a first come, first served basis.

\subsubsection{Physical Therapy}

An email blast was sent to second year students in Physical Therapy requesting volunteers to participate in the project.

\subsubsection{Master of Health Administration}

Students in their second year of a two-year program were given the opportunity to submit an application for selection into the IPE student cohort. Participation was voluntary and was not associated with an existing course nor was course credit provided. Interested students submitted a one-paragraph statement that addressed the following: (1) why the applicant desired to participate in the program and (2) how participation would align with the applicant's career goals. All applicants who provided a well-reasoned and supported application were selected.

\subsection{Health Professional Faculty}

The development and implementation of the educational experiences was labor-intensive for faculty who, though appointed by their Deans, served as volunteers. As is the case in many university settings, there were no incentives (e.g., course release or overload pay) for faculty to assume additional responsibilities related to IPE. The initiative required sustained effort in recruitment of students, supervision of online activities, as well as planning and implementing over three years to achieve the project objectives. Six faculty developed the educational experiences and, though supported in the implementation by the seven-member faculty practice team, were responsible for the educational initiative. Anecdotal reports from nationally recognized projects note the difficulty in sustaining faculty volunteers in interprofessional education over the long-term. However, in our case, the faculty worked together over the three-year period and there was no attrition.

\subsection{Scheduling}

Scheduling was a major challenge in recruiting students and proved difficult as each health professional program has unique academic schedules. For example, nursing consists of a two and half year program while pharmacy and optometry are four years without summer interruption in courses. Some programs follow a traditional fall and spring semester while others deliver curricula in a modular integrated format (e.g., in the school of pharmacy, the cardiovascular module runs through 8 weeks in the spring, while the endocrine occurs in the fall for 5 weeks). Most programs offer courses during the day while the MHA program delivers all of its courses during the evening. Combined, these differences made it difficult to schedule a time that students and faculty could meet face-to-face. It was found that face-to-face sessions were better suited for students and faculty during the early evening at the beginning of the week. The IPE educational experience schedule prepared by IPE faculty and shared with students in advance allowed for a 90\%-95\% attendance at each face-to-face session.

\subsection{Sustainability of IPE: The importance of a shared definition of IPE}

Internal and external factors including geography, accreditation standards, tuition differentials and longevity of programs have influenced the ability to achieve a shared vision and shared definition of IPE at the administrative levels of the University. Although the grant received from the Division of Nursing of HRSA had initial support from the Deans representing the five programs, unexpected programmatic and administrative changes within the schools exposed differences in the understanding of interprofessional education and collaborative practice within and across programs. In some cases, Deans who initially were enthusiastic during the grant-writing and submission phases modified the extent of their commitment and student involvement as the realities of project implementation, such as scheduling, unfolded. However, the Deans of Nursing and Pharmacy, who were under accreditation requirements for the specific inclusion of IPE, maintained support over the long-term.

To manage these issues, the definition of interprofessional education gravitated from one that included the collaboration of all five health professional disciplines to one put forth by the World Health Organization (World Health Organization [WHO], 2010). Interestingly, faculty who were directly engaged in the project embraced the WHO definition from the beginning of the initiative which reads as follows: "when students from two or more professions learn about, from and with each other to enable effective collaboration and improve health outcomes" 
(WHO, 2010). This clear definition presupposes work on the IPE competencies and includes clinical practice. However, a major task for university administration is to formally define IPECP at the Deans level, which would serve as a guide for current IPECP endeavors and foster collaboration across the health professions and future programs. This may require additional time together, engaging in discussions of program goals, roles and responsibilities as well as participating in exercises to build trust that promote interprofessional communication no matter the geographical location.

\section{Discussion}

We learned a number of lessons from the experience of integrating interprofessional education into the curricula of five health professional programs.

(1) A large number of students from each of the professions were willing to volunteer time and effort, often without credit, to learn about interprofessional collaborative care.

(2) A viable model of education for the university has been developed. This model consisted of a blended learning approach including online and face-to-face interactions. The blended approach assisted in mitigating the difficulties associated with scheduling and timing.

(3) The use of interactive teaching strategies, particularly high fidelity simulation, demonstrated to students the value of an interprofessional approach to patient care and allowed for good participation from faculty and students.

(4) Faculty who participated in IPE increased their knowledge about IPE, embraced the concepts of interprofessionality quickly, demonstrated creativity in developing interactive educational strategies, and maintained a sustained engagement over a three-year period.

Differences in accreditation requirements across programs are a barrier to full participation by program administrators and have the potential to inhibit development of a shared University-wide definition of IPE. In our case, not all health professional school's accrediting agencies have provided a definition or guidelines of an acceptable IPE experience for faculty and students. New programs require sustained attention to curricular detail and often lack the flexibility necessary to test implementation of new experiences such as interprofessional didactic and clinical experiences. A careful analysis of the readiness of programs with recognition of the importance of timing for success may be more important to appropriate program inclusivity in the early stages of a university's trajectory.

In summary, interprofessional education and collaborative practice is not a new paradigm to be considered and evaluated. Instead, it is a necessary practice given the imperative for change in the current US health system. Health professional schools and the university in which they reside will need additional resources and infrastructure support in order to successfully integrate and sustain IPE programs. A deeper understanding of IPE by university administration, the necessary time and commitment to revise courses and the provision of resources for faculty development on IPE course creation and delivery requires engagement from the highest level of university administration to assure collaboration across programs and adequate resourcing. More than anything else, university administrators must understand that the investment in this important endeavor goes beyond meeting the requirements of accreditation. Effective and sustainable interprofessional education will provide health professional students the knowledge and skills necessary to be collaborative practice-ready - an essential competency in the increasingly patient-centered, cost sensitive, quality-focused, world of healthcare reform.

\section{Acknowledgements}

This project is/was supported by the Health Resources and Services Administration (HRSA) of the U.S. Department of Health and Human Services (HHS) under grant number UD7HP25055-02-01 Division of Nursing, Health Resources and Human Services Health Profession, Nurse Education, Practice, Quality and Retention (NEPQR) ProgramInterprofessional Collaborative Practice- Award; 1.3 million over 3 years. This information or content and conclusions are those of the author and should not be construed as the official position or policy of, nor should any endorsements be inferred by HRSA, HHS or the U.S. Government.

\section{References}

Accreditation Council on Optometric Education. (2009). Professional optometric degree standards. Retrieved from: http://www.aoa.org/Documents/students/od_standards_effective_jan_1_2009.doc

Accreditation Council for Pharmacy Education. (2011). Accreditation standards and guidelines for the professional program in pharmacy leading to the doctor of pharmacy degree. Retrieved from: https://www.acpe-accredit.org/pdf/FinalS2007Guidelines2.0.pdf 
Benner, P. (2011). Formation in professional education: An examination of the relationship between theories of meaning and theories of the self. Journal Of Medicine \& Philosophy, 36(4), 342-353. http://dx.doi.org/10.1093/jmp/jhr030

Cauthon, K. A., Nguyen, C. N., Ramirez, M. N., Ybarra, L. M., Parker, R. A., \& Jones, M. E. (2015). Snapshot of the impact of interprofessional collaboration in practice in diabetes care. American Association of Diabetes Educators (AADE) in Practice, 3(3): 20-29. http://dx.doi.org/10.1177/2325160315574591

Commission on Accreditation of Healthcare Management Education. (2013). Fall 2013 CAHME criteria for accreditation. Retrieved from: http://www.cahme.org/Resources/Fall2013_CriteriaForAccreditation.pdf

Commission on Accreditation of Physical Therapy Education. (2013). Evaluative criteria for accreditation of education programs for the preparation of physical therapists. Retrieved from: http://www.capteonline.org/AccreditationHandbook/

D’Amour, D. \& Oandasan,, I. (2005). Interprofessionality as the field of interprofessional practice and interprofessional education: An emerging concept. Journal of Interprofessional Care. 19(1), 18-20. http://dx.doi.org/10.1080/13561820500081604

Institute of Medicine. (1972). Educating for the health team: Report of the conference on the interrelationships of educational programs for health professionals. Washington, DC: National Academy of Sciences.

Interprofessional Education Collaborative Expert Panel. (2011). Core competencies for interprofessional collaborative practice. Washington, DC: Interprofessional Education Collaborative. Retrieved from: http://www.aacn.nche.edu/education-resources/ipecreport.pdf

Josiah Macy Foundation. (2013). Transforming patient care: Aligning interprofessional education with clinical practice design. Atlanta, Georgia: Josiah Macy Foundation. Retrieved from: http://macyfoundation.org/docs/macy_pubs/JMF_TransformingPatientCare_Jan2013Conference_fin_Web.pdf

Lapkin, S., Levett-Jones, T. \& Gilligan, C. (2013). A systematic review of the effectiveness of interprofessional education in health professional programs. Nurse Education Today, 33(2), 90-102. http://dx.doi.org/10.1016/j.nedt.2011.11.006

Robert Wood Johnson Foundation. (2011). Advancing interprofessional education. Retrieved from: http://www.rwjf.org/en/library/articles-and-news/2011/05/advancing-interprofessional-education.html

Thistlethwaite, J. (2012). Interprofessional education: A review of context, learning and the research agenda. Medical Education, 46(1), 58-70. http://dx.doi.org/10.1111/j.1365-2923.2011.04143.x

World Health Organization. (2010). Framework for action on interprofessional education and collaborative practice, Geneva, Switzerland: World Health Organization Department of Human Resources for Health. 